\title{
Enhancing Face Recognition Performance using Triplet Half Band Wavelet Filter Bank
}

\author{
Mohd.Abdul Muqeet \\ Muffakham Jah College of Engineering and Technology, Hyderabad, India \\ Email: ab.muqeet2013@gmail.com \\ Raghunath S.Holambe \\ SGGS Institute of Engineering and Technology, Nanded, India \\ Email:holambe@yahoo.com
}

\begin{abstract}
Face recognition using subspace methods are quite popular in research community. This paper proposes an efficient face recognition method based on the application of recently developed triplet half band wavelet filter bank (TWFB) as pre-processing step to further enhance the performance of well known linear and nonlinear subspace methods such as principle component analysis(PCA),kernel principle component analysis (KPCA), linear discriminant analysis (LDA), and kernel discriminant analysis (KDA). The design of $6^{\text {th }}$ order TWFB is used as the multiresolution analysis tool to perform the 2-D discrete wavelet transform (DWT). Experimental results are performed on two standard databases ORL and Yale. Comparative results are obtained in terms of verification performance parameters such as false acceptance rate (FAR), false rejection rate (FRR) and genuine acceptance rate (GAR). Application of TWFB enhances the performance of PCA, KPCA, LDA, and KDA based methods.
\end{abstract}

Index Terms-Face Recognition, triplet half band wavelet filter bank (TWFB), PCA, KPCA, LDA, KDA.

\section{INTRODUCTION}

Face recognition has rapidly developed over past of the years with important applications in video surveillance identity authentication, security monitoring, access control, suspect tracking in commercial and law enforcement [1].Challenges in face recognition arise due to change in face image characteristics such as a change in illumination of scene, change in pose, change in facial expression, and occlusion of some portion of the face area. Appearance-based face recognition methods are categorized into linear and nonlinear subspace methods and frequency based methods. Principal component analysis (PCA) [3], Fisher discriminant analysis (FDA) [4], independent component analysis (ICA) [5] and twodimensional PCA [6] are the widely used linear subspace methods. These methods attempt to represent face images in a lower dimensional feature space which is a linear combination of a set of basis [25]. In spite of the popularity of linear subspace methods, their performance is not satisfactory under large variation of facial images such as illumination pose and occlusion. To overcome such variations some nonlinear subspace methods like kernel PCA (KPCA) [7] and kernel discriminant analysis (KDA) [8] are proposed which are non linear extension of PCA and LDA. These methods map an input image nonlinearly to a higher dimensional feature with the help of a kernel function [8]. Non-statistical face recognition method using local binary pattern (LBP) [26] outperforms PCA and LDA-based methods in terms of recognition performance and computational simplicity. Disadvantages are sensitivity to noise and a large size of the feature vector. Wavelet transforms based face recognition methods are extensively by the research community. Researchers have used different off the shelf wavelet filter for facial feature extraction. This paper involves the usage of recently developed triplet wavelet filter bank (TWFB) to enhance the performance of existing linear and non linear subspace methods. Application of (TWFB) provides the efficient multiresolution features compared with the existing wavelet filters. The paper is organized as follows. Section II gives the insight of the related work. In Section III, we briefly reviewed linear and non linear subspace methods such as PCA [3], LDA [4], KPCA [7], and KDA [8]. Section IV reviews the design of TWFB [19], [22]. The proposed method of face recognition using TWFB based features, used face databases, and experimental results are summarized in Section $\mathrm{V}$ followed by conclusion in Section VI.

\section{RELATED WORK}

Recent progress in face recognition efficiently uses wavelet transform that possess good time and frequency localization which helps to detect the facial geometric structures and offers robust feature extraction even under variable illumination [9]. In wavelet analysis the discrete wavelet transform (DWT) [10] and Gabor wavelet transform (GWT) [11]-[12] based face recognition methods are more popular. GWT is more successful due to its capability of effectively expressing face feature for its directional selectivity. But Gabor wavelets provide over-complete representation which increases computational complexity due to the convolution of each 
face image with several Gabor wavelet kernels at many scales and orientations [13]. When DWT is applied before PCA or LDA, features like edge information are extracted more efficiently which forms meaningful feature vectors [13].Such a method proposed by Feng et al [14] applied PCA on wavelet subband and utilized a midrange frequency subband for PCA representation. Chien and $\mathrm{Wu}$ [10] performed face recognition by applying the wavelet transform to extract waveletfaces from face images and incorporated LDA for discriminant analysis. A comparison of the performance of face recognition systems based on the principal component analysis (PCA), Gabor wavelet transform (GWT) and discrete wavelet transform (DWT) is presented in [15]. It is demonstrated that the DWT algorithm has the ability to achieve recognition performance at levels similar to those of the GWT algorithm with a faster analysis time. Utsumi et al [9] evaluated the performance of face recognition in response to various wavelets transform (e.g. Haar, French hat, Mexican hat, Daubechies, Coiflet, Symlet, and Ospline).They demonstrated that the performance of the wavelets assessed is similar to that of the Gabor wavelet. Jadhav and Holambe [16] applied radon transform to images and DWT is applied on the generated radon feature space. Radon transform improves the lowfrequency component of the face images and wavelet transform when applied on the Radon feature space provides multiresolution features. The proposed system is invariant in facial expression and illumination. Most of the existing DWT based face extraction methods use offthe-shelf wavelet filters like orthogonal (Haar, Daubechies, Coiflet, Symlet) and Biorthogonal (B-spline Biorthogonal, and CDF-9/7). But even for the same class of face images, the effect of these wavelet filters may vary for the distinct application. This is due to the fact that many of the face images do not have the same statistical characteristics due to the changes in illumination, pose, and occlusion. Designing of appropriate wavelet filters can result in robust representations with regard to changes in illumination, pose, and occlusion and provide significant feature vectors with reduce computational complexity. Hence to design wavelet filters which match the characteristics of face images for feature extraction is highly desirable. Biorthogonal wavelet filters are preferred over orthogonal wavelet filters for applications such as feature extraction and image compression. The most popular construction for the design of biorthogonal filter banks is CDF-9/7 9/7 [17] which is designed by factorizing a Lagrange half band polynomial (LHBP) to obtain wavelet filters in the filter bank. Lagrange half band polynomial is imposed with maximum no of zeros at $z=-1$ due to which they offer no degree of freedom and there is no direct control over the frequency response of the filters [19],[22]. Patil et al [18] designed a pair of biorthogonal wavelet filters to control the frequency response of the filters. In their proposed work instead of using the Lagrange half band polynomial a general half band polynomial (GHBP) is considered for factorization. A recent work on designing of biorthogonal wavelet filter bank is proposed by
Rahulkar et.al [19], [22].A general half band polynomial is used and three polynomials are generated using factorization. These triplet filters are used in the equations proposed in [21] to obtain the low pass analysis and synthesis filters. The authors used it for Iris feature extraction, however due to its properties like flexible frequency response, near-Orthogonality, and regularity; the designed filter bank can be effectively used for applications in feature extraction and image compression [22].The authors in [23] used the 10th order TWFB for iris feature extraction.

Our work is motivated from [19] and [22] to develop a face recognition system so as to derive significant and compact facial features using the TWFB as the preprocessing step and provide the essential multiresolution features to the existing subspace methods like PCA, KPCA, LDA, and KDA. Experiments are performed on two well known databases ORL [27] and Yale [28].

\section{OVERVIEW OF LINEAR AND NON-LINEAR SUB-SPACE METHODS}

In this section, a brief review on linear and non-linear sub-space methods is presented.

\section{A. Principal Component Analysis}

Principal component analysis is a famous linear projection method for dimensionality reduction [3]. Assuming the training set consists of $\mathrm{N}$ images, the data matrix $X=\left\{x_{1}, x_{2}, \ldots . x_{N}\right\}, x_{i} \in R^{t}$ is obtained by row concatenation of image data. Let $C$ be the number of classes and each image $x_{i}$ belongs to one of $C$ classes $\{1,2, \ldots \ldots . C\}$.The mean face of the data matrix is defined as $m=(1 / N) \cdot \sum_{i=1}^{N} x_{i}$. The covariance matrix or the total scatter matrix $S_{t}$ is defined as [3]:

$$
S_{t}=\frac{1}{N} \sum_{i=1}^{N}\left(x_{i}-m\right) \cdot\left(x_{i}-m\right)^{T}
$$

The eigenvalues $\lambda_{i}$ and corresponding eigenvectors $V_{i}$ are computed from $S_{t}$ defined by the following eigenvalues problem [3]:

$$
S_{t} V_{i}=\lambda_{i} V_{i}
$$

Arranging all eigenvalues in the descending order and considering first few $n$ highest eigenvalues and their corresponding eigenvectors generates the projection matrix $W_{p c a}$ and in turn new face space [3],

$$
Z=W_{p c a}^{T}\left(x_{i}-m\right), \text { where } z_{i} \in R^{f}(f<<t)
$$

\section{B. Linear Discriminant Analysis (LDA)}


The objective of Fisherfaces method using linear discriminant analysis (LDA) is to choose subspace of face features that makes the ratio of between-class scatter matrix to the within-class scatter matrix largest [4]. For all samples of all classes, the between-class scatter matrix $S_{b}$ and the within-class scatter matrix $S_{w}$ are defined as [4]:

$$
\begin{gathered}
S_{b}=\sum_{i=1}^{C} n_{i} \cdot\left(m_{i}-m\right) \cdot\left(m_{i}-m\right)^{T} \\
S_{w}=\sum_{i=1}^{C} \sum_{j=1}^{n_{i}}\left(x_{j}-m_{i}\right)\left(x_{j}-m_{i}\right)^{T}
\end{gathered}
$$

Where $n_{i}$ is the number of samples in ith class $m_{i}$ is the mean for the ith class samples and $m$ is the mean face or global mean of all samples. If $S_{w}$ is non-singular, LDA seeks to find the optimal transformation matrix $W$ to maximize the projection ratio denoted by [4]:

$$
J(W)=\arg \max _{W} \frac{\left|W^{T} S_{b} W\right|}{\left|W^{T} S_{w} W\right|}
$$

This ratio is maximized when $W$ is constructed by considering $C-1$ leading eigenvectors of the following eigenvalue problem [4]:

$$
S_{w}^{-1} S_{b} w_{i}=\lambda_{i} w_{i} \quad i=1,2, \ldots \ldots, C-1
$$

Where $w_{i}(i=1,2, \ldots \ldots, C-1) \quad$ is the generalized eigenvectors corresponding to the largest eigenvalues.

But $S_{w}$ is always singular in face recognition due to the high dimensionality of face images [4]. Thus to make the $S_{w}$ matrix non-singular PCA is usually performed before LDA to reduce the dimensions from $N$ to $N-C$ and then applying LDA defined by equation (6) to further reduce the dimension to $C-1$ [4].

\section{Kernel principal component analysis (KPCA)}

KPCA is a nonlinear extension of classical PCA [7]. The mapping of linear input space $R^{t}$ into high dimensional feature space $F$ is obtained by a nonlinear mapping function,

$$
\begin{gathered}
\phi: R^{t} \rightarrow F \\
x \mapsto \phi(x)
\end{gathered}
$$

This non linear mapping is achieved though kernels trick [7]. The covariance matrix on the feature space $F$ is:

$$
S_{t}^{\phi}=\frac{1}{N} \cdot \sum_{i=1}^{N}\left(\phi\left(x_{i}\right)-m_{0}^{\phi}\right)\left(\phi\left(x_{i}\right)-m_{0}^{\phi}\right)^{T}
$$

where

$$
m_{0}^{\phi}=\frac{1}{N} \cdot \sum_{i=1}^{N} \phi\left(x_{i}\right)
$$

The corresponding eigen-value problem becomes [7]:

$$
S_{t}^{\phi} V_{i}=\lambda_{i} V_{i}
$$

Applying the kernel trick, equation (11) can be transformed to

$$
K \tilde{V}_{i}=\tilde{\lambda}_{i} \tilde{V}_{i}, \quad \text { for } \quad i=1, \ldots . N
$$

$K$ is the $N \times N$ centralized kernel matrix defined with

$$
K_{i j}=\phi\left(x_{i}\right)^{T} . \phi\left(x_{j}\right)=\left(\phi\left(x_{i}\right) \bullet \phi\left(x_{j}\right)\right)=k\left(x_{i}, x_{j}\right)
$$

where $k\left(x_{i}, x_{j}\right)$ is a kernel function employed to compute the dot product $\left(\phi\left(x_{i}\right) . \phi\left(x_{j}\right)\right)$ and $(i, j=1, \ldots ., N)$

Thus calculating the orthonormal eigenvectors $\tilde{V}_{1}, \tilde{V}_{2}, \ldots \ldots, \tilde{V}_{n}$ corresponding to $n$ largest positive eigenvalues $\tilde{\lambda}_{1}, \tilde{\lambda}_{2}, \ldots . . \tilde{\lambda}_{n}$, the new face space is calculated using [7].

$$
Y_{i}=\sum_{i=1}^{n} \frac{\tilde{V}_{i}}{\sqrt{\tilde{\lambda} i}} \cdot k\left(x_{i}, x\right)
$$

\section{Kernel Discriminant Analysis(KDA)}

In the proposed work of KDA [8] the kernel feature space $F$ is used instead of input space $R^{t}$ to find the traditional LDA. The between and within-class scatter matrix in feature space $F$ are as

$$
S_{b}^{\phi}=\sum_{i=1}^{C} n_{i}\left(m_{i}^{\phi}-m^{\phi}\right)\left(m_{i}^{\phi}-m^{\phi}\right)^{T}
$$

and

$$
S_{w}^{\phi}=\sum_{i=1}^{C} \sum_{j=1}^{n_{i}}\left(\phi\left(x_{j}\right)-m_{i}^{\phi}\right)\left(\phi\left(x_{j}\right)-m_{i}^{\phi}\right)^{T}
$$

where $m_{i}^{\phi}$ is the mean of $\mathrm{i}$-th class mapping samples,$m^{\phi}$ is the is the global mean of all the mapping samples and $n_{i}$ is the number of samples in i-th class.

Fisher discriminant function in $\mathrm{F}$ feature space is given as [8]:

$$
J_{1}(w)=\frac{w^{T} S_{b}^{\phi} w}{w^{T} S_{w}^{\phi} w}
$$


Since eigenvectors of $w$ lies in the span of all samples in $F$, its eigenvectors can be represented with a linear combination of $\phi\left(x_{i}\right)$, i.e.

$$
w=\sum_{i=1}^{N} a_{i} \phi\left(x_{i}\right)=Q a
$$

Substituting equation (18) in equation (17), we obtain the following maximizing function for KDA [8]

$$
J_{2}(w)=\frac{a^{T} K_{b} a}{a^{T} K_{w} a}
$$

Thus from solution of equation (19) we obtain the eigenvectors corresponding to the largest eigen-values in the following eigen-value problem:

$$
K_{w}^{-1} K_{b} \alpha_{i}=\lambda_{i} \alpha_{i}
$$

For $n$ largest eigen-values we use the following formula to calculate projection of a new sample $x$ on $w$ in $F$ feature space

$$
y_{i}=(w . \phi(x))=\alpha_{i}^{T} K_{x}=\sum_{i=1}^{n} \alpha_{i} k\left(x_{i}, x\right)
$$

\section{TRIPLET HALF BAND WAVELET FILTER BANK (TWFB) AND FEATURE EXTRACTION}

\section{A. Triplet half band wavelet filter bank (TWFB): Design Review}

As per the design methodology adopted in [19],[22],[23] only FIR filters for implementation of a two channel biorthogonal channel filter bank shown in Fig.1, where $H_{0}(z)$ and $H_{1}(z)$ are the low pass and high pass filters respectively. The corresponding $G_{0}(z)$ and $G_{1}(z)$ are the low pass and high pass synthesis filters. The perfect reconstruction condition [20] is given as

$$
G_{0}(z) H_{0}(z)+G_{1}(z) H_{1}(z)=2 z^{-D}
$$

and

$$
G_{0}(z) H_{0}(-z)+G_{1}(z) H_{1}(-z)=0
$$

Where $\mathrm{D}$ is the amount of delay. If $H_{1}(z)=G_{0}(-z)$ and $G_{1}(z)=-H_{0}(-z)$ then equation (2) is automatically satisfied and equation (1) reduces to equation (3) by defining the product filter $P(z)=H_{0}(z) \cdot G_{0}(z)$. Thus, the design of the filter bank reduces to the design of the half band filter $P(z)$ whose factorization gives $H_{0}(z)$ and $G_{0}(z)$ [20].

$$
P(z)-P(-z)=2 z^{-D}, \text { where } \mathrm{D} \text { is odd }
$$

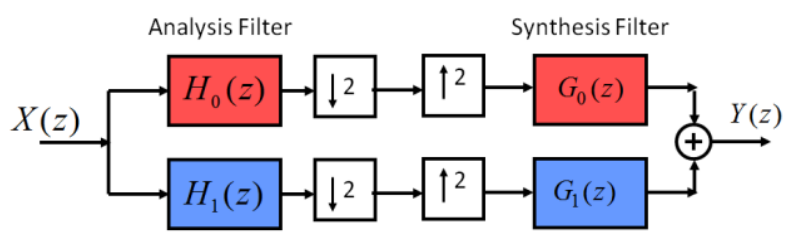

Fig.1. Two-channel Biorthogonal Filter Bank

As per the work described in [19], [22], [23], a $6^{\text {th }}$ order generalized half band polynomial is used to design $(13 / 19)$ wavelet filters. The design procedure for the triplet half band wavelet filter bank (TWFB) is explained as below

1) Consider a generalized half band polynomial (GHBP) $P(z)$ of $6^{\text {th }}$ order i.e. $K=6$.

$$
P(z)=\alpha_{0}+\alpha_{2} z^{-2}+z^{-3}+\alpha_{2} z^{-4}+\alpha_{0} z^{-6}
$$

2) This GHBP is used to construct three half band polynomials (HBPs) by extracting three zeros at $z=-1$ using the method of synthetic division [19], [22]. Extracting of zeros at $\mathrm{z}=-1$ imposes regularity on the GHBP $P(z)$ and should satisfy the constraints of $X<((K / 2)+1)$, where $\mathrm{X}$ are the number of zeros at $z=-1$ and $K$ is the order of GHBP.

3) Regularity is imposed in the design of $P(z)$ by extracting three zeros at $\mathrm{z}=-1$ to construct three half band polynomials $P_{1}(z), P_{2}(z), P_{3}(z)$.These three half band polynomials are expressed in terms of $\alpha_{0}$ only which are given as follows [19],[22],[23]

$$
\begin{aligned}
& P_{1}(z)=\left(1+z^{-1}\right)^{X_{1}} \cdot R_{1}(z) \\
& P_{2}(z)=\left(1+z^{-1}\right)^{X_{2}} \cdot R_{2}(z) \\
& P_{3}(z)=\left(1+z^{-1}\right)^{X_{3}} \cdot R_{3}(z)
\end{aligned}
$$

Where $X_{i}$ are number of zeros at $\mathrm{z}=-1$, and $R_{1}(z), R_{2}(z), R_{3}(z)$ the remainder polynomials.

Consider $X_{1}=0, X_{2}=1$ and $X_{2}=2$ such that $X=X_{1}+X_{2}+X_{3}=3$ which satisfies the condition of $X<((K / 2)+1), K=6$ is the order of GHBP. 


$$
\begin{aligned}
& P_{1}(z)=(1+z)^{0} \cdot\left(\alpha_{0}+\left(-\alpha_{0}+0.5\right) z^{-2}+z^{-3}\right. \\
& \left.+\left(-\alpha_{0}+0.5\right) z^{-4}+\alpha_{0} z^{-6}\right) \\
& P_{2}(z)=\left(1+z^{-1}\right)^{1} \cdot\left(\alpha_{0}-\alpha_{0} z^{-1}+0.5 z^{-2}\right. \\
& \left.+0.5 z^{-3}-\alpha_{0} z^{-4}+\alpha_{0} z^{-5}\right) \\
& P_{3}(z)=\left(1+z^{-1}\right)^{2} \cdot\left(\alpha_{0}-2 \alpha_{0} z^{-1}+\left(2 \alpha_{0}+0.5\right) z^{-2}(27)\right. \\
& \left.-2 \alpha_{0} z^{-3}+\alpha_{0} z^{-4}\right)
\end{aligned}
$$

As these HBPs are expressed in the form of $\alpha_{0}$ term, only a flexible frequency response can be obtained due to one degree of freedom [19], [22]. The value of $\alpha_{0}=-0.062499$ is obtained using MATLAB unconstrained optimization function fminunc which considers the objective function designed on the basis of minimizing energy in the ripples of these three HBPs[19],[22].

4) The analysis low pass $H_{0}(z)$ and synthesis low pass $G_{0}(z)$ are given as follows from [19] and [21]

$$
\begin{gathered}
H_{0}(z)=\frac{1+p}{2}+\frac{1}{2} T_{1}(z) \cdot\left(1+p T_{0}(z)\right) \\
G_{0}(z)=\frac{1+p T_{0}(z)}{1+p}+\frac{1-p}{1+p} T_{2}(z) \cdot H_{0}(-z)
\end{gathered}
$$

Where $T_{0}(z), T_{1}(z)$ and $T_{2}(z)$ are half band kernels whose ideal pass band and stop band responses are 1 and 0 respectively. In order to achieve perfect reconstruction, these filters are formulated as below.

$$
\begin{aligned}
& T_{0}(z)=P_{1}(z)-1 \\
& T_{1}(z)=P_{2}(z)-1 \\
& T_{2}(z)=P_{3}(z)-1
\end{aligned}
$$

5) Substituting equation (29) in equation (28) we can obtain low pass analysis $H_{0}(z)$ and low pass synthesis $G_{0}(z)$ filters of TWFB.

To obtain same magnitude response from $H_{0}(z)$ and $G_{0}(z)$ at $\omega=\pi / 2$, the value of shaping parameter $p=0.41421$ is considered [21], [22]. The length of $H_{0}(z)$ and $G_{0}(z)$ is 13 and 19 respectively.

6) By using the below relation we can obtain the high pass analysis and synthesis filters.

$$
G_{0}(z)=H_{1}(-z), G_{1}(z)=-H_{0}(-z)
$$

Thus, the usage of a GHBP to design TWFB improves the frequency response of $H_{0}(z)$ and $G_{0}(z)$ filters. These filters not only satisfy regularity but also achieve linear phase and perfect reconstruction [19], [22]. These properties are vital in achieving higher facial discrimination capability along with linear and non linear subspace methods.

\section{B. Facial Feature Extraction Using TWFB}

We need 2-D separable filter bank for face feature extraction and we need only analysis or decomposition part of the filter bank. The implementation is carried out by applying one-dimensional wavelet filters to the rows of the face image and the columns of the row transformed data respectively. The one level-decomposition results in one approximation subband $L L$ which gives a coarser approximation to the original image, one $L H$ band and one $H L$ band which records the changes of the image along the horizontal and vertical direction, and one $H H$ band that shows the high-frequency component of the image. In our approach, TWFB is applied on face images to extract multiresolution-based facial feature. First, we perform two-level wavelet decomposition using the TWFB wavelet pairs and obtain the two-level lowfrequency sub-band $L L$ as the feature vectors. The lowfrequency subband $L L$ is an optimal approximation image of the original image which contains most important features of the face and can be sufficiently used for face recognition and the remaining high-frequency sub-bands can be neglected. These wavelet pairs provide an effective discriminatory representation of face images. Thus, after obtaining the multiresolution facial features using the TWFB, linear and non linear subspace methods are used to obtain the enhanced face recognition performance. To capture the difference between the two facial features or classification, we used Euclidean distance measures for LDA and KDA based methods and cosine distance measures for PCA and KPCA based face recognition methods.

\section{EXPERIMENTATION AND RESULTS}

The experiments were carried out in Matlab 2014a, on a 64-bit I3, $2.13 \mathrm{GHz}$ processor, with $2 \mathrm{~GB}$ RAM. The performance evaluation is carried out on two standard databases, ORL [27], and Yale [28] face databases. To design the experiments for face verification we consider all the face images and applied the concept mentioned in previous section for performance measure. In this paper, to test the robustness of the proposed approach no preprocessing technique is used for face images such as face normalization. Thus to enhance the performance of PCA, LDA, KPCA, and KDA, 2-D implementation of TWFB is applied on the face images. Compared with the linear and non subspace projection methods which take raw pixel information, TWFB facial features contains more discriminating information thus shows robustness against variation in illumination, expression, and pose.

\section{A. Performance Measures}

The verification performance of our face recognition methods is estimated using false acceptance rate (FAR); false reject rate (FRR) and guanine GAR values. FAR is 
defined as the ratio of the number of accepted impostors to the total number of impostor accesses, while FRR is defined as the ratio of rejected genuine claims to the total number of genuine accesses. Where impostors are defined as the face images for an individual excluding his own images. A low value for FAR and FRR is often desirable but as the FAR increases FRR decreases, this is due to the uneven distribution of genuine and impostor claims. Thus to determine the optimum balance between FAR and FRR, Receiver Operating Curve (ROC) is adopted which plot FAR versus GAR [24].A higher value of GAR is often desirable for a better verification performance.

\section{B. Results on ORL face database}

The ORL database [27] consists of 10 different images of each of 40 different persons. There are variations in the capture time, lighting, head position, facial expressions such as eyes open or closed, smiling or not smiling and facial details such as glasses or no glasses. All images are 8-bit grayscale taken against a dark homogeneous background of resolution $112 \times 92$ pixels. The face images are resized to $128 \times 128$ pixels. Some samples face images of ORL face database are shown in Fig.2. The first series of experiments are conducted using the extracted TWFB features and four subspace methods for face verification. For this purpose, all parameters like feature dimensions and distance measure are optimized to achieve the best performance.

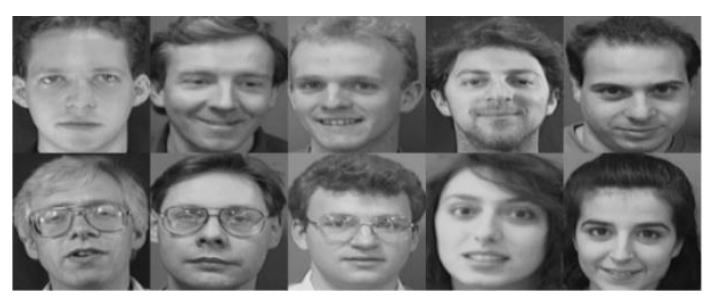

Fig.2. Sample face images from ORL face database

Comparative results of PCA, LDA, KPCA and KDA are tabulated in Table. 1 and same is shown with ROC curves in Fig. 3. For these methods, the pixel values of face images are simply concatenated to form a feature vector. For KPCA and KDA based methods, we adopted normalized polynomial kernel with the parameter $d$ equal to 2 .

Table 1. Sub-space results in terms of FAR, FRR, and GAR on ORL database

\begin{tabular}{|c|c|c|c|}
\hline Method & FAR $(\%)$ & FRR $(\%)$ & GAR $(\%)$ \\
\hline PCA & 0.9744 & 3.2727 & 96.7273 \\
\hline LDA & 0.6957 & 1.8636 & 98.1364 \\
\hline KPCA & 0.4169 & 3.4545 & 96.5455 \\
\hline KDA & 0.2558 & 1.8182 & 98.1818 \\
\hline
\end{tabular}

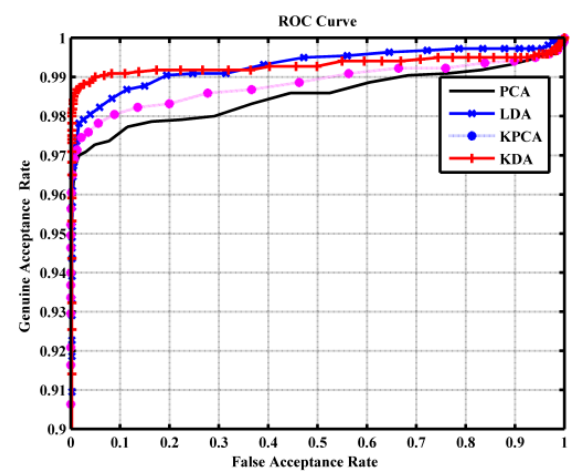

Fig.3. ROC curves of the subspace methods for ORL database

To verify the discriminating power of extracted TWFB based pixel features over the raw pixels of subspace methods a comparative performance of $\mathrm{TWFB}+\mathrm{PCA}$, TWFB+ LDA, TWFB +KPCA and TWFB+KDA is carried out. It can be observed from Table. 2 and Fig.4 that the adaptation of TWFB before PCA, LDA, KPCA, and KDA methods enhances the performance of these methods and we can achieve better performance compared to the original methods. The dimension of the feature vector for PCA and KPCA based methods is 399, whereas its 39 for LDA and KDA based methods. The great advantage of applying TWFB comes from the fact that the size for the low frequency approximation subband of size $32 \times 32$ is suffice to capture the essential discriminating characteristics of face image compared to original image size of $128 \times 128$ as in case with PCA,LDA,KPCA and KDA methods.

LDA-based method gives better performance than PCA-based method and kernel-based methods give better performance than linear-based PCA and LDA methods. Among all these methods, the KDA based methods combined with TWFB gives the best performance in terms of higher GAR when the dimension is set at 39 .

Table 2. TWFB based Sub-space results in terms of FAR, FRR, and GAR on ORL database

\begin{tabular}{|c|c|c|c|}
\hline Method & FAR (\%) & FRR (\%) & GAR (\%) \\
\hline TWFB+PCA & 0.3031 & 1.3182 & 98.6818 \\
\hline TWFB+LDA & 0.7161 & 1.2273 & 98.7727 \\
\hline TWFB+KPCA & 0.3510 & 1.9545 & 98.0455 \\
\hline TWFB+KDA & 0.2558 & 1.1364 & 98.8636 \\
\hline
\end{tabular}

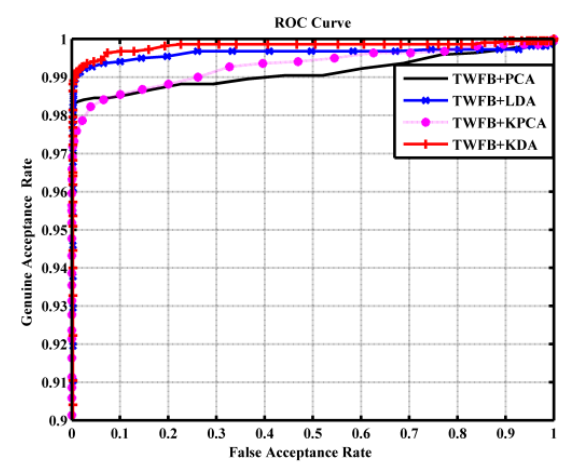

Fig.4. ROC curves of the TWFB based subspace methods on ORL database 
It is apparent from Table. 2 and Fig. 4 that adaptation of TWFB based feature vectors enhances the performance of these subspace methods with difference in GAR. TWFB+PCA method achieves $2.02 \%$ higher GAR compared with PCA, while $0.6442 \%$ higher GAR is observed for TWFB+LDA compared with LDA method. For TWFB+KPCA and for TWFB+KDA, $1.53 \%$ and $0.6944 \%$ higher GAR is achieved respectively.

\section{Results on Yale face Database}

Yale face database [28] consists of face 15 individuals and for each individual there are 11 images. Images are having variations in illumination and facial expressions. The illumination variations are due to lighting changes in center-light, left-light and right-light. Different facial expressions are normal, happy, sad, sleepy, surprise and wink. We used this database to evaluate the performance of our method under the condition of change in illumination and facial expression. The face images are resized to $128 \times 128$ pixels. Samples face images of one person are shown in Fig.5.

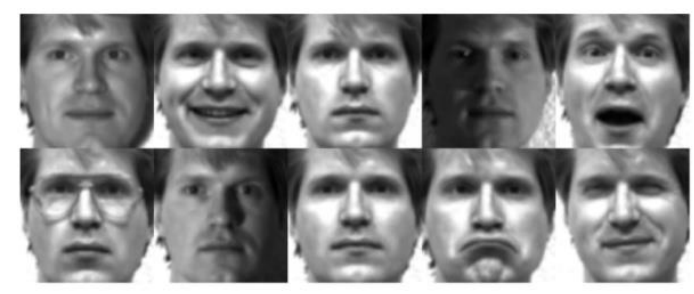

Fig.5. Sample face from Yale face database with different illumination variation

Similar FAR and FRR test are performed on Yale database which gives 990 genuine attempts and 25575 impostor attempts. The performance values attained with the method taking raw pixel values images applying only PCA, LDA, KPCA and KDA are tabulated in Table. 3 and the corresponding ROC curve is shown in Fig.6.

Table 3. Subspace results in terms of FAR, FRR, and GAR on Yale face database

\begin{tabular}{|c|c|c|c|}
\hline Method & FAR (\%) & FRR (\%) & GAR (\%) \\
\hline PCA & 6.9169 & 27.1717 & 71.6162 \\
\hline LDA & 0.6452 & 3.3333 & 96.6667 \\
\hline KPCA & 6.6276 & 27.1717 & 72.8283 \\
\hline KDA & 0.6452 & 1.5152 & 98.4848 \\
\hline
\end{tabular}

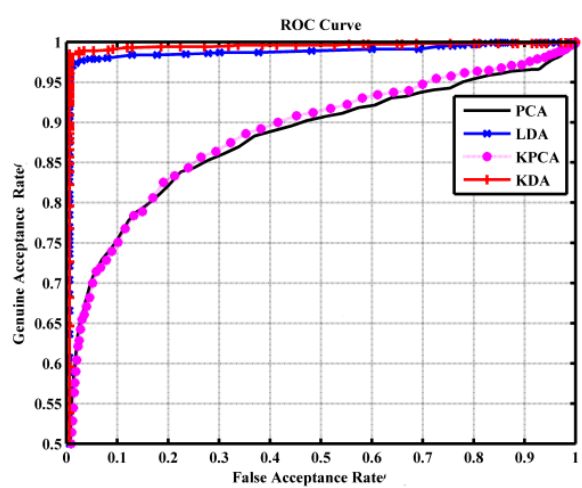

Fig.6. ROC curves of the subspace methods on Yale database
TWFB based sup-space methods are tabulated in Table 4 and shown in Fig.7. It is evident that KPCA achieves better performance compared with PCA and KDA achieve better performance compared with LDAbased method for Yale database. For Yale databases, TWFB+PCA method achieves $0.28 \%$ higher GAR compared with PCA, while $0.42 \%$ higher GAR is observed for TWFB+LDA compared with LDA method. For TWFB+KPCA $1.39 \%$ and for TWFB+KDA $0.6154 \%$ higher GAR is achieved respectively.

Table 4. TWFB based Subspace results in terms of FAR, FRR, and GAR on Yale database

\begin{tabular}{|l|l|l|l|}
\hline Method & FAR $(\%)$ & FRR $(\%)$ & GAR $(\%)$ \\
\hline TWFB-+PCA & 4.2972 & 28.1818 & 71.8182 \\
\hline TWFB+LDA & 1.2551 & 2.9293 & 97.0707 \\
\hline TWFB+KPCA & 10.5298 & 26.1616 & 73.8384 \\
\hline TWFB+KDA & 0.6452 & 0.9091 & 99.0909 \\
\hline
\end{tabular}

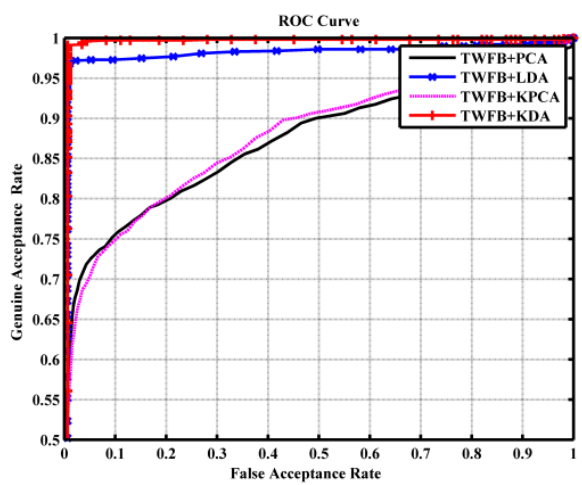

Fig.7. ROC curves of the TWFB based subspace methods on Yale database

\section{Comparative results}

We also compared the effectiveness of TWFB as facial features with CDF-9/7 wavelet filters, as these filters are mostly used for wavelet based facial feature extraction. The comparative results are depicted in Table 5.

Table 5. Comparative results

\begin{tabular}{|l|l|l|l|l|l|l|}
\hline \multirow{2}{*}{ Method } & \multicolumn{6}{|l|}{ ORL } \\
\cline { 2 - 7 } & $\begin{array}{l}\text { FAR } \\
(\%)\end{array}$ & $\begin{array}{l}\text { FRR } \\
(\%)\end{array}$ & $\begin{array}{l}\text { GAR } \\
(\%)\end{array}$ & $\begin{array}{l}\text { FAR } \\
(\%)\end{array}$ & $\begin{array}{l}\text { FRR } \\
(\%)\end{array}$ & $\begin{array}{l}\text { GAR } \\
(\%)\end{array}$ \\
\hline $\begin{array}{l}\text { CDF-9/7+ } \\
\text { LDA }\end{array}$ & 0.514 & 1.318 & 98.682 & 1.255 & 2.929 & 97.071 \\
\hline TWFB+LDA & 0.716 & 1.227 & 98.773 & 1.857 & 2.323 & $\mathbf{9 7 . 6 7 7}$ \\
\hline $\begin{array}{l}\text { CDF-9/7+ } \\
\text { KDA }\end{array}$ & 0.302 & 1.500 & 98.500 & 0.645 & 1.111 & 98.889 \\
\hline TWFB+KDA & 0.255 & 1.136 & 98.864 & 0.645 & 0.909 & $\mathbf{9 9 . 0 9 1}$ \\
\hline
\end{tabular}

\section{CONCLUSION}

In this paper, we have proposed a face recognition method to enhance the performance of existing subspace methods with application of TWFB. The design of TWFB for facial feature extraction is also been discussed and experimentally tested for face verification purpose. The performance evaluation is carried out on two standard 
face databases. The proposed method with TWFB+KDA achieve high GAR values of $98.86 \%$ and $99.09 \%$ method for ORL and Yale face databases respectively, which is significantly better than PCA, LDA, and KPCA based methods.

\section{REFERENCES}

[1] W.Zhao,R Chellappa, A.Rosenfeld, and P.J.Phillips, "Face Recognition: A Literature Survey", Technical Report CAR-TR-948,Univ. of Maryland, CfAR, (2000).

[2] S. Z. Li and A. K. Jain, Handbook of Face Recognition, eds. Springer-Verlag, Mar.2005

[3] M. Turk and A. Pentland, "Eigenfaces for recognition," $J$. Cogn. Neurosci., vol. 3, no. 1, pp. 71-86, 1991

[4] P. Belhumeur, J. Hespanha, and D. Kriegman, "Eigenfaces vs Fisherfaces: Recognition using class specific linear projection," IEEE Trans. Pattern Anal. Mach. Intell., vol. 20, no. 7, pp. 711-720, Jul. 1997

[5] M. Bartlett, J. Movellan, and T. Sejnowski, "Face recognition by independent component analysis," IEEE Trans. Neural Netw., vol. 13, pp. 1450-1464, 2002

[6] J. Yang, D. Zhang, A.F. Frangi, and J.Y. Yang, "Twodimensional PCA: A new approach to appearance-based face representation and recognition," IEEE Trans. Pattern Anal. Mach. Intell., vol.26, no.1, pp.131-137, Jan. 2004

[7] K.-R. Müller, S. Mika, G. Rätsch, K. Tsuda, and B. Schölkopf, "An introduction to kernel-based learning algorithms," IEEE Trans. Neural Networks, vol. 12, pp. 181-201, Mar. 2001.

[8] J. W. Lu, K. Plataniotis, and A. N. Venetsanopoulos, "Face recognition using kernel direct discriminant analysis algorithms," IEEE Trans. Neural Netw., vol. 14, no. 1, pp. 117-126, Jan. 2003.

[9] Y. Utsumi, Y. Iwai, and M. Yachida, "Performance evaluation of face recognition in the wavelet domain," in Proc. Int. Con. Intelligent Robots and Systems, 2006, pp. 3344-3351.

[10] J. T. Chien and C. C.Wu, "Discriminant waveletfaces and nearest feature classifiers for face recognition," IEEE Trans. Pattern Anal. Mach Intell., vol. 24, no. 12, pp. 1644-1649, Dec. 2002.

[11] C. J. Liu and H.Wechsler, "Gabor feature based classification using the enhanced fisher linear discriminant model for face recognition," IEEE Trans. Image Process., vol. 11, no. 4, pp. 467-476, Apr. 2002

[12] Štruc V., Pavešic, N, "The Complete Gabor-Fisher Classifier for Robust Face Recognition," EURASIP Advances in Signal Processing, vol. 2010, 26 pages, doi: $10.1155 / 2010 / 847680,2010$

[13] L. L. Shen and L. Bai, "A review on gabor wavelets for face recognition," Pattern Anal. Appl., vol. 9, pp. 273-292, 2006

[14] G. C. Feng, P. C. Yuen and D. Q. Dai, "Human face recognition using PCA on wavelet subband," Journal of Electronic Imaging, 9(2), 2000, pp. 226-233

[15] M. Meade. S.C.Sivakumar,W. J. Phillips," Comparative performance of principal component analysis, gabor wavelets and discrete wavelet transforms for face recognition," Electrical and Computer Engineering, Canadian Journal, vol.30, no.2, pp.93,102, Spring 2005 doi: 10.1109/CJECE.2005.1541731

[16] D.V. Jadhav, R.S. Holambe, "Feature extraction using Radon and Wavelet transforms with application to face recognition," Journal of Neurocomputing 72 (2008) 1951-1959.
[17] C. I. Daubechies and J. C. Feauveau, "Biorthogonal bases of compactly supported wavelets," Commun. Pure Appl. Math., vol. 45, no. 5, pp. 485-560, 1992.

[18] B. D. Patil, P. G. Patwardhan, and V. M. Gadre, "On the design of FIR wavelet filter banks using factorization of a halfband polynomial," IEEE Signal Process. Lett., vol. 15, pp. $485-488,2008$

[19] A. D. Rahulkar and R. S. Holambe, "Half-iris feature extraction and recognition using a new class of biorthogonal triplet half-band filter bank and flexible kout-of-n: A postclassifier," IEEE Trans. Inf. Forensics Security, vol. 7, no. 1, pp. 230-240, Feb. 2012

[20] G. Strang and T. Nguyen, Wavelets and Filter Banks. Cambridge, MA: Wellesley-Cambridge, 1996.

[21] R. Ansari, C. W. Kim, and M. Dedovic, "Structure and design of two channel filter banks derived from a triplet of halfband filtres," IEEE Trans. Circuits Syst. II, Analog Digit. Signal Process., vol. 46, no. 12, pp. 1487-1496, Dec. 1999.

[22] Rahulkar A.D, Patil B.D, Holambe R.S, "A new approach to the design of biorthogonal triplet half-band filter banks using generalized half-band polynomials", Signal Image Video Process 2012: 1-7. http://dx.doi.org/10.1007/s11760-012-0378-1.

[23] S.S.Barpanda, B.Majhi, P.K.Sa, "Region based feature extraction from non-cooperative iris images using triplet half-band filter bank," Optics and Laser Technology, 72 (2015)6-14

http://dx.doi.org/10.1016/j.optlastec.2015.03.003

[24] K. Jonsson, J. Kittler, Y.P. Li, J. Matas, "Support vector machines for face authentication," Image and Vision Computing 20 (5-6) (2002) 369-375.

[25] Ali Javed, -Face Recognition Based on Principal Component Analysis, I.J. Image, Graphics and Signal Processing (IJIGSP), 2013, 2, 38-44. DOI: 10.5815/ijigsp.2013.02.06

[26] Murty, Gorti Satyanarayana, J. Sasi Kiran, and V. Vijaya Kumar. "Facial Expression Recognition Based on Features Derived From the Distinct LBP and GLCM." International Journal of Image, Graphics and Signal Processing, vol.6, no. 2, pp.68, 2014.

[27] [Online].Available: http://www.uk.research.att.com/pub/data/att_faces.zip

[28] [Online].Available: http://cvc.yale.edu/projects/yalefaces/yalefaces.html.

\section{Authors' Profiles}

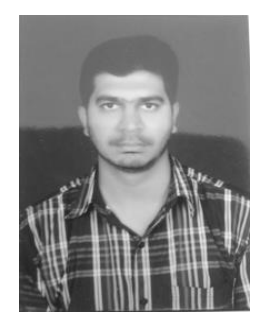

Mohd.Abdul Muqeet, received the B.E. degree from M.B.E.S College of Engineering, Ambejogai, India in 2001, and M.Tech. degree from SGGS Institute of Engineering and Technology, Nanded, India in 2007 . He is presently a research scholar in Instrumentation Engineering at SGGS Institute of Engineering and Technology, Nanded, India. His research interests include filter banks, biometrics, and applications of wavelet transform. 


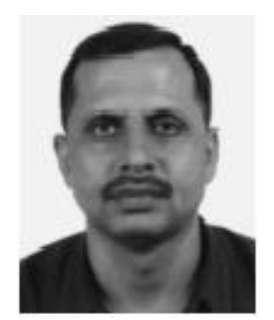

Dr. Raghunath S. Holambe received the Ph.D. degree from Indian Institute of Technology, Kharagpur, India, and he is presently a professor in Instrumentation Engineering in SGGS Institute of Engineering and Technology, Nanded, India. The areas of his research interest are digital signal processing, image processing, applications of wavelet transform, biometrics, and real time signal processing using DSP processors.

How to cite this paper: Mohd.Abdul Muqeet, Raghunath S.Holambe,"Enhancing Face Recognition Performance using Triplet Half Band Wavelet Filter Bank", International Journal of Image, Graphics and Signal Processing(IJIGSP), Vol.8, No.12, pp.62-70, 2016.DOI: 10.5815/ijigsp.2016.12.08 